\title{
Erratum to: Elaboration of a nomogram to predict nonsentinel node status in breast cancer patients with positive sentinel node, intraoperatively assessed with one step nucleic amplification: Retrospective and validation phase
}

\author{
Franco Di Filippo ${ }^{1 *}$, Simona Di Filippo ${ }^{2}$, Anna Maria Ferrari ${ }^{3}$, Raffaele Antonetti ${ }^{4}$, Alessandro Battaglia ${ }^{5}$, \\ Francesca Becherini ${ }^{6}$, Laia Bernet ${ }^{7}$, Renzo Boldorini ${ }^{8}$, Catherine Bouteille ${ }^{9}$, Simonetta Buglioni ${ }^{1}$, Paolo Burelli ${ }^{6}$, \\ Rafael Cano ${ }^{10}$, Vincenzo Canzonieri ${ }^{11}$, Pierluigi Chiodera ${ }^{12}$, Alfredo Cirilli ${ }^{13}$, Luigi Coppola ${ }^{14}$, Stefano Drago ${ }^{14}$, \\ Luca Di Tommaso ${ }^{15}$, Privato Fenaroli ${ }^{16}$, Roberto Franchini ${ }^{17}$, Andrea Gianatti ${ }^{16}$, Diana Giannarelli ${ }^{1}$, \\ Carmela Giardina $^{18}$, Florence Godey ${ }^{19}$, Massimo M. Grassi ${ }^{20}$, Giuseppe B. Grassi ${ }^{14}$, Siobhan Laws ${ }^{21}$, \\ Samuele Massarut ${ }^{11}$, Giuseppe Naccarato ${ }^{22}$, Maria Iole Natalicchio ${ }^{23}$, Sergio Orefice ${ }^{24}$, Fabrizio Palmieri ${ }^{25}$, \\ Tiziana Perin ${ }^{11}$, Manuela Roncella ${ }^{26}$, Massimo G. Roncalli ${ }^{27}$, Antonio Rulli ${ }^{28}$, Angelo Sidoni ${ }^{28}$, Corrado Tinterri ${ }^{27}$, \\ Maria C. Truglia ${ }^{29}$ and Isabella Sperduti ${ }^{1}$
}

\section{Erratum}

Upon publication of the original article [1], it was noticed that the affiliation of the authors Vincenzo Canzonieri, Tiziana Perin and Samuele Massarut, "Centro di Riferimento Oncologico NCI IRCCS - Aviano" was incorrectly given as "Centro Regionale Oncologico". This has now been acknowledged and corrected in this erratum.

\footnotetext{
Author details

${ }^{1}$ Regina Elena National Cancer Institute, Via Elio Chianesi 53, 00144 Rome, Italy. ${ }^{2}$ Ospedale di Latina, Latina, Italy. ${ }^{3}$ San Camillo, Milan, Italy. ${ }^{4} \mathrm{Az}$. Ospedaliera Universitaria Foggia, Foggia, Italy. ${ }^{5} \mathrm{ASL}$, Prato, Italy. ${ }^{6}$ ULSS 7 Pieve di Soligo, Pieve di Soligo, Italy. ${ }^{7}$ Hospital Lluís Alcanyís, Xàtiva, Spain.

${ }^{8}$ Università of Piemonte, Vercelli, Italy. ${ }^{9}$ Hôpitaux de Lyon, Lyon, France.

${ }^{10}$ Hospital Universitario de La Ribera, Alzira, Spain. ${ }^{11}$ Centro di Riferimento Oncologico NCI IRCCS - Aviano, Bari, Italy. ${ }^{12}$ San Donato, Italy. ${ }^{13}$ Policlinico of Bari, Bari, Italy. ${ }^{14}$ San Filippo Neri, Florence, Italy. ${ }^{15}$ Humanitas Rozzano, Rozzano, Italy. ${ }^{16}$ ASST Papa Giovanni XXIII, Bergamo, Italy. ${ }^{17}$ Azienda Ospedaliera "Maggiore della Carità" di Novara, Novara, Italy. ${ }^{18}$ University of Bari, Bari, Italy. ${ }^{19}$ Eugene Marquis Cancer Center, Rennes, France. ${ }^{20}$ Humanitas Gavazzeni, Bergamo, Italy. ${ }^{21}$ Hampshire Hospitals NHS Foundation Trust, England, UK. ${ }^{22}$ University of Pisa, Pisa, Italy. ${ }^{23}$ Azienda

Ospedaliero-Universitaria OO.RR. Foggia, Foggia, Italy. ${ }^{24}$ University of L'Aquila, L'Aquila, Italy. ${ }^{25}$ I.R.C.C.S. L. Spallanzani, Rome, Italy. ${ }^{26}$ Azienda
}

\footnotetext{
*Correspondence: franco.difilippo@ifo.gov.t; franco.difilippo49@gmail.com
${ }^{1}$ Regina Elena National Cancer Institute, Via Elio Chianesi 53, 00144 Rome,

*Correspondence: franco.difilippo@ifo.gov.it; franco.difilippo49@gmail.com
${ }^{1}$ Regina Elena National Cancer Institute, Via Elio Chianesi 53, 00144 Rome, Italy
}

Ospedaliero-Universitaria Pisana, Pisa, Italy. ${ }^{27}$ Humanitas, Pisa, Italy.
${ }^{28}$ University of Perugia, Perugia, Italy. ${ }^{29}$ USL, Prato, Italy.

Received: 10 May 2017 Accepted: 10 May 2017

Published online: 16 May 2017

\section{Reference}

1. Di Filippo F, Di Filippo S, Ferrari AM, Antonetti R, Battaglia A, Becherini F, Bernet L, Boldorini R, Bouteille C, Buglioni S, Burelli P. Elaboration of a nomogram to predict nonsentinel node status in breast cancer patients with positive sentinel node, intraoperatively assessed with one step nucleic amplification: Retrospective and validation phase. J Exp Clin Cancer Res. 2016;35(1):193. 